\title{
BENEFICIOS DE LA HIDROTERAPIA EN EL TRABAJO DE PARTO. ESTUDIO DE REVISIÓN
}

Andrea Benavides Navarro ${ }^{1}$

Correo electrónico: andrea17_39@hotmail.com

1. Enfermera en el Hospital General de L’Hospitalet.

Recibido: 11-12-2019 Aceptado: 03-01-2020

(c) (i) (2) 


\section{RESUMEN}

\section{Introducción}

La reciente demanda de las gestantes para lograr un parto lo más fisiológico posible ha propiciado una disminución de las técnicas intervencionistas y consecuentemente un auge en la búsqueda de técnicas alternativas para el alivio del dolor durante el trabajo de parto, entre las que podemos destacar la hidroterapia.

\section{Objetivos}

Describir la eficacia del uso de la hidroterapia en relación con la percepción del dolor durante el trabajo de parto.

\section{Metodología}

Revisión bibliográfica en la que se han utilizado las bases de datos PubMed, Cuiden, Cuidatge, Biblioteca Cochrane y CINHAL.

\section{Resultados}

Se han incluido un total de 17 artículos: 13 artículos originales y 4 revisiones bibliográficas. Además, se ha utilizado la Guía de Práctica Clínica sobre la Atención al Parto Normal. Los artículos hacen referencia a los resultados maternos y perinatales del uso de la hidroterapia en la fase de dilatación y la fase expulsiva, así como las percepciones de las enfermeras, matronas y gestantes sobre el uso de esta novedosa técnica.

\section{Conclusiones}

La hidroterapia utilizada durante la fase de dilatación del parto disminuye la percepción del dolor y el uso de medidas farmacológicas. Sin embargo, la seguridad y la eficacia de la inmersión en agua en la fase expulsiva no han sido establecidas y no se han asociado beneficios maternales o fetales.

Palabras clave: parto; hidroterapia; terapias complementarias; dolor. 


\section{ABSTRACT}

\section{Introduction}

The growing demand of pregnant women to achieve a delivery as physiological as possible has led to a decrease in interventional techniques and consequently a rise in the search for alternative techniques for the relief of pain during labor, among which we can highlight hydrotherapy.

\section{Objectives}

To describe the effectiveness of the use of hydrotherapy in relation to the perception of pain during labor.

\section{Methodology}

Bibliographic review using the databases PubMed, Cuiden, Cuidatge, Cochrane Library and CINHAL.

\section{Results}

A total of 17 articles have been included: 13 original articles and 4 bibliographic reviews. In addition to this, the Clinical Practice Guideline on Normal Birth Care has been used. The articles refer to the maternal and perinatal results of the use of hydrotherapy in the dilatation phase and the expulsive phase, as well as the perceptions of midwives, nurses and pregnant women of its use.

\section{Conclusions}

The use of the hydrotherapy during the dilation phase of labor decreases the perception of pain and the use of pharmacological measures. However, the safety and efficacy of immersion in water in the expulsive phase have not been established and no benefits have been associated for the mother or the foetus.

Keywords: Birth; Hydrotherapy/Waterbirth/Bathpool; Complementary Therapies; Pain. 


\section{INTRODUCCIÓN}

El parto y el nacimiento son experiencias profundas y únicas y, al mismo tiempo, procesos fisiológicos complejos (Ministerio de Sanidad y Política Social, 2010). En las últimas décadas se ha producido un cambio conceptual, tanto en la asistencia obstétrica como en la asistencia al trabajo de parto. Este cambio tiene como eje fundamental que la gestante sea el centro de la asistencia recibida. Esto es debido a que cada vez más las gestantes buscan tener un parto con una atención que sea tan fisiológica como sea posible y en el cual se eviten intervenciones innecesarias (Sociedad Española de Obstetricia y Ginecología, 2008).

La gestante tiene el derecho a decidir qué asistencia desea para su parto, siempre con la máxima seguridad y en el lugar que ella elija, facilitando así una experiencia de nacimiento más positiva y feliz para ella y su bebé. Por lo tanto, es necesario ofrecer la atención, la información, el asesoramiento y el acompañamiento adecuado durante todo el proceso (Federación de Asociaciones de Matronas de España, 2017).

La atención al parto intervencionista ha ido perdiendo fuerza delante de una nueva filosofía de entender el desarrollo y la atención sanitaria al parto, propiciando una rápida expansión en el desarrollo y el uso de un amplio abanico de prácticas alternativas ideadas para controlar todos los aspectos del proceso del parto con el propósito de propiciar una mejora en el desenlace para las madres y sus bebés (Ministerio de Sanidad y Política Social, 2010).

\section{Parto normal o eutócico}

A pesar del considerable debate y los estudios realizados a lo largo de los años, el concepto de «normalidad» en el parto y en el nacimiento no está estandarizado ni universalizado (Organización Mundial de la Salud, 1996). El criterio de que el parto solo puede considerarse normal de forma retrospectiva ha llevado, junto con otros factores, a proporcionar a los partos normales cuidados muy semejantes a los que necesitan los partos complicados, transformando un proceso fisiológico en un procedimiento médico quirúrgico (Ministerio de Sanidad y Política Social, 2010).

Podemos encontrar diversas definiciones de lo que se considera parto normal, una de estas definiciones es la realizada por la Sociedad Española de Ginecología y Obstetricia (SEGO) que lo definió como: «Trabajo de parto de una gestante sin factores de riesgo durante la gestación, que se inicia de forma espontánea entre las 37-42 semanas y que tras 
una evolución fisiológica de la dilatación y el parto, termina con el nacimiento de un recién nacido normal que se adapta de forma adecuada a la vida extrauterina. El alumbramiento y el puerperio inmediato deben, igualmente, evolucionar de forma fisiológica» (Sociedad Española de Obstetricia y Ginecología, 2008).

Otra definición es la de FAME (Federación de Asociaciones de Matronas de España) en el año 2007, la cual dice: «Es el proceso fisiológico único con el que la mujer finaliza su gestación a término, en el que están implicados factores psicológicos y socioculturales. $\mathrm{Su}$ inicio es espontáneo, se desarrolla y termina sin complicaciones, culmina con el nacimiento y no implica más intervención que el apoyo integral y respetuoso del mismo». También la Guía de práctica clínica sobre la atención al parto normal del Ministerio de Sanidad y Consumo del año 2010 describe: «Un parto normal es aquel con un comienzo espontáneo, que presenta un bajo riesgo al comienzo y que se mantiene como tal hasta el alumbramiento. El niño o niña nace espontáneamente en posición cefálica entre las semanas 37 a 42 completas. Después de dar a luz, tanto la madre y su bebé se encuentran en buenas condiciones».

\section{El dolor en la fisiología del parto}

El parto es descrito por muchas mujeres como uno de los momentos más dolorosos que experimentan en sus vidas. Ello ha propiciado durante décadas la búsqueda de estrategias analgésicas, tanto farmacológicas como no farmacológicas para el alivio del dolor, como puede ser el uso de la hidroterapia durante el trabajo de parto (Ministerio de Sanidad y Política Social, 2010).

Se define el dolor de parto como «experiencia sensitivo-perceptual y emocional única, influida por múltiples factores internos y externos, que se activa como respuesta fisiológica a un estímulo orgánico producido por las contracciones uterinas y la expulsión del feto y los anejos, cuyo componente es tanto físico como psicológico-emocional».

Los diversos aspectos que interaccionan en su aparición, transmisión y vivencia hacen que realizar una definición concreta de dolor de parto sea muy complejo (Mallén-Pérez et al., 2015). El dolor durante el trabajo de parto supone una gran preocupación para muchas mujeres y la mayoría requiere algún tipo de alivio del dolor. 


\section{Antecedentes del uso de la hidroterapia durante la fase expulsiva del parto}

Existen documentos que relatan el uso de la hidroterapia como método para aliviar el dolor en las culturas romanas, griegas y egipcias, pero no se sabe con exactitud cuándo empezó su uso realmente. La primera vez que se relató el parto de una mujer con un bebe sano nacido en una bañera fue en 1805, a manos de Embry M., pero no fue hasta 1983 cuando se pudo hacer difusión de la técnica gracias a Odent M. (1999: 37-41), ya que fue el primero en introducir en la unidad de maternidad del Hospital de Pithiviers de Francia, una bañera de partos inflable como estrategia para reducir el uso de drogas analgésicas durante el parto (Mallén-Pérez, 2017)

Sin embargo, hasta 1993 no se produjo una aceptación oficial del uso de la hidroterapia como método para aliviar el dolor durante el proceso de dilatación en el parto gracias a la publicación en el Reino Unido del informe «Changing Childbirth». Un año después, el Royal College of Midwifes y el United Kingdom Central Council for Nursing, Midwifery and Health Visiting publicaron un documento en el cual se posicionaban a favor del uso de agua caliente durante el parto para aliviar el dolor (Royal College of Midwives, 2009).

Actualmente este método tiene su máxima expansión y adhesión en el Reino Unido, país pionero en emplear esta técnica, dónde más del 95\% de los servicios de maternidad poseen una bañera a disposición de las gestantes que lo deseen (Burns et al., 2012). No obstante, la situación en los demás países desarrollados está en continuo crecimiento desde las últimas décadas, debido a la creciente demanda de las gestantes para tener un parto lo más fisiológico posible (Nicholls S et al. 2016).

En Catalunya la demanda de esta modalidad ha aumentado en los últimos años, lo que ha llevado a los organismos oficiales a promover la implantación de este nuevo método en los centros de maternidad de la comunidad autónoma (Generalitat de Catalunya, 2013).

Por tanto, se puede constatar que desde los inicios de este método hasta la actualidad ha habido un aumento en el estudio e implantación de la hidroterapia como método para aliviar el dolor durante el trabajo de parto.

\section{El rol de enfermeras y matronas}

Virginia Henderson afirmaba que es una función básica de cualquier enfermera propiciar el desarrollo de la autonomía del paciente y que sea él o ella quién decida cómo quiere enfocar sus cuidados (Bellido et al., 2010). 
Es de gran necesidad que las futuras madres conozcan las diferentes alternativas que hay para dar a luz, y es precisament en el momento de proporcionar información cuando las enfermeras pueden contribuir aportando información sobre el amplio abanico de alternativas que existen, siempre basándose en la evidencia científica, y con ello ayudar a que, gracias a esta información, sean las propias mujeres quienes decidan como desean enfocar su futuro parto y la atención sanitaria al mismo.

Se puede observar un gran incremento en la demanda de nuevas alternativas naturales al parto ya que cada vez más las mujeres buscan un parto que sea lo más fisiológico posible y en el cual se eviten intervenciones innecesarias (Burns et al., 2012). Por este motivo, los profesionales deben impregnarse de esta realidad y estudiar las diferentes alternativas que existen para así garantizar la autonomía y el total bienestar de la mujer durante el parto. Sin embargo, la gran mayoría de profesionales desconocen el tema o simplemente han oído hablar de ello.

Debido a que existen controversias en cuanto a su uso, y teniendo en cuenta que la hidroterapia ha llegado a ser uno de los métodos alternativos más utilizados, surge la siguiente pregunta: ¿Existen beneficios en cuanto al uso de la hidroterapia durante el trabajo parto, diferenciando sus dos etapas: la fase de dilatación y la fase expulsiva?

Teniendo todas estas premisas en cuenta, el presente trabajo pretende abordar el siguiente objetivo: Conocer la eficacia del uso de la hidroterapia en relación con la percepción del dolor durante el proceso de dilatación.

\section{METODOLOGÍA}

Revisión de la bibliografía científica publicada con el fin de conocer y describir la eficacia del uso de la hidroterapia frente al dolor durante el trabajo de parto. En la Tabla 1 se detallan las palabras clave utilizadas para la búsqueda de información. 
Tabla 1. Palabras clave

\begin{tabular}{|l|l|l|}
\hline \multicolumn{1}{|c|}{ Castellano } & \multicolumn{1}{c|}{ Catalán } & \multicolumn{1}{c|}{ Inglés } \\
\hline Hidroterapia & Hidroteràpia & $\begin{array}{l}\text { Hydrotherapy/Waterbirth/ } \\
\text { Bathpool }\end{array}$ \\
\hline Parto & Part & Delivery \\
\hline Terapias complementarias & Teràpies complementàries & Complementary therapies \\
\hline Dolor & Dolor & Pain \\
\hline Agua & Aigua & Water \\
\hline Efectividad & Efectivitat & Effectiveness \\
\hline
\end{tabular}

En la Tabla 2 se especifican las bases de datos usadas, así como los términos, booleanos y límites establecidos, los resultados hallados y los artículos seleccionados para realizar la revisión bibliográfica.

Se han empleado como fuentes de información las bases de datos CINAHL, Pubmed, Cuidatge, CUIDEN y Cochrane Library. También se ha utilizado el Descriptor en Ciencias de la Salud (DeCS) para traducir correctamente las palabras clave utilizadas para la búsqueda de información. Se han utilizado los booleanos «AND»y «OR», así como los siguientes límites: año de publicación 2008-2018, texto en inglés, catalán o castellano y relacionado con el ser humano.

Durante la búsqueda de información, se decidió ampliar el año de publicación de 5 a 10 años, ya que así se disponía artículos de mayor calidad para la revisión.

Los criterios de inclusión han sido los siguientes: artículos que estudien a seres humanos publicados entre los años 2008 y 2018, siendo los idiomas de estos inglés, castellano o catalán. No se han incluido aquellos artículos que no hagan referencia explícita al objetivo planteado, así como tampoco aquellos artículos que no diferenciasen si el uso de la hidroterapia se aplicaba en la primera o en la segunda fase del parto.

Una vez realizada la búsqueda en las bases de datos, se han seleccionado, en una primera revisión, aquellos artículos cuyo título sea específico sobre el tema a tratar. Seguidamente, se ha procedido a leer el resumen de estos artículos.

De los 49 artículos encontrados, se seleccionaron un total de 17 artículos, debido a duplicidades o no adecuarse a los objetivos pactados. También se descartaron diversas 
revisiones bibliográficas, ya que para llevar a cabo este estudio interesaba nueva documentación científica que avalara el uso de la hidroterapia durante las diferentes fases del parto.

Tabla 2. BBDD, términos, booleanos y límites, resultados y artículos seleccionados

\begin{tabular}{|c|c|c|c|}
\hline Bases de datos & Términos, booleanos y límites & Resultados & $\begin{array}{l}\text { Artículos } \\
\text { seleccionados }\end{array}$ \\
\hline \multirow{2}{*}{ CINHAL } & $\begin{array}{l}\text { Hydrotherapy } \\
\text { Límites: } \\
\text { Fecha de publicación 20080101-20181231, } \\
\text { • Humano, Idioma English, Spanish y } \\
\quad \text { Embarazo }\end{array}$ & 18 & 10 \\
\hline & $\begin{array}{l}\text { Hydrotherapy AND (labor and delivery) AND } \\
\text { (pain relief or pain management). } \\
\text { Límites: Fecha de publicación 20080101- } \\
20181231\end{array}$ & 6 & 5 \\
\hline \multirow{2}{*}{$\begin{array}{l}\text { COCHRANE } \\
\text { LIBRARY }\end{array}$} & $\begin{array}{l}\text { Hydrotherapy } \\
\text { Límites: Año de publicación desde } 2008 \text { hasta } \\
2018 \text { (se han buscado variaciones de palabras) }\end{array}$ & 69 & 1 \\
\hline & $\begin{array}{l}\text { Hydrotherapy AND pain AND labor } \\
\text { Límites: Año de publicación desde } 2008 \text { hasta } \\
2018 \text { (se han buscado variaciones de palabras) }\end{array}$ & 5 & 3 \\
\hline \multirow{2}{*}{ CUIDEN } & Hidroterapia & 77 & 9 \\
\hline & Hidroterapia AND parto & 13 & 8 \\
\hline CUIDATGE & Agua AND parto & 13 & 7 \\
\hline PubMed & $\begin{array}{l}\text { Waterbirth } \\
\text { Límites: Año de publicación } 2008 \text { hasta } 2018 . \\
\text { Idiomas inglés y castellano }\end{array}$ & 47 & 6 \\
\hline TOTAL & & 248 & 49 \\
\hline
\end{tabular}

\section{RESULTADOS}

A continuación, se presenta un resumen de los 17 artículos seleccionados clasificados según su uso en las diferentes fases del parto (Tabla 3), o según la percepción de la utilidad de la hidroterapia por enfermeras o matronas (Tabla 4). 
Tabla 3. Resultados maternos y perinatales en relación con el uso de la hidroterapia en las diferentes fases del parto

\begin{tabular}{|c|c|c|c|c|c|}
\hline Autor, Año, País & $\begin{array}{c}\text { Tipo } \\
\text { articulo/ } \\
\text { Diseño }\end{array}$ & Sujetos/Ámbito & $\begin{array}{l}\text { Variables/Dimensiones/ } \\
\text { Instrumentos/Intervención }\end{array}$ & Resultados & $\begin{array}{l}\text { Conclusiones/Implicaciones } \\
\text { práctica/investigación }\end{array}$ \\
\hline $\begin{array}{c}\text { Mollamahmutoğlu et al. } \\
\text { Turquía } \\
2011\end{array}$ & $\begin{array}{l}\text { AO, Quant., } \\
\text { Exp. }\end{array}$ & $\begin{array}{l}\text { Selección de } 602 \text { mujeres: } \\
207 \text { parto acuático, } 204 \\
\text { parto convencional y } 191 \\
\text { con uso de la epidural. }\end{array}$ & $\begin{array}{l}\text { Duración del primer, segundo y } \\
\text { tercer período del parto, } \\
\text { requerimientos de inducción y } \\
\text { episiotomía, traumatismos } \\
\text { perineales y puntuaciones de } \\
\text { Apgar. }\end{array}$ & $\begin{array}{l}\text { La duración de la } 1^{\mathrm{a}} \text { fase del parto fue } \\
\text { menor en los partos acuáticos que en los } \\
\text { convencionales y epidural, pero la } 2^{\mathrm{a}} \text { y } 3^{\mathrm{a}} \\
\text { fase fueron proporcionalmente incluso de } \\
\text { menor duración en partos acuáticos en } \\
\text { relación con partos convencionales y con } \\
\text { epidural. Las gestantes que realizaron un } \\
\text { parto en el agua mostraron: menos } \\
\text { requerimientos de inducción, episiotomía } \\
\text { y analgesia, pero tuvieron más laceración } \\
\text { perineal. }\end{array}$ & $\begin{array}{l}\text { Ventajas nacimiento en el agua: } \\
\text { Reducción en la duración de la } 2^{\text {o }} \text { y } \\
3^{\circ} \text { etapa del parto, reducción del } \\
\text { dolor y la intervención obstétrica } \\
\text { como la inducción o la } \\
\text { episiotomía. Contribuye } \\
\text { positivamente a la salud fisiológica } \\
\text { y psicológica de la madre. No hay } \\
\text { aumento de los efectos adversos } \\
\text { para el feto o las gestantes. }\end{array}$ \\
\hline $\begin{array}{c}\text { Ros } \\
2009 \\
\text { Sudáfrica }\end{array}$ & $\begin{array}{l}\text { AO, Des., } \\
\text { Exp. }\end{array}$ & $\begin{array}{l}\text { Recién nacidos de madres } \\
\text { que dieron a luz en la sala } \\
\text { de partos de un hospital del } \\
\text { gobierno y en dos unidades } \\
\text { de nacimiento en hospitales } \\
\text { privados en Gauteng. }\end{array}$ & $\begin{array}{l}\text { Explorar y describir los } \\
\text { resultados perinatales en partos } \\
\text { con inmersión acuática (grupo A) } \\
\text { y partos convencionales (grupo } \\
\text { B). Se analizaron los siguientes } \\
\text { resultados perinatales: } \\
\text { puntuación de Apgar al minuto y } \\
\text { a los 5', Ta axilar, pH, } \\
\text { hemoglobina y niveles de sodio } \\
\text { de la sangre del cordón } \\
\text { umbilical, condición neurológica } \\
\text { del recién nacido plasmada en los } \\
\text { reflejos primitivos y la } \\
\text { morbilidad neonatal hasta } 14 \text { días } \\
\text { después del nacimiento. }\end{array}$ & $\begin{array}{l}\text { El grupo A tenía puntuaciones de Apgar } \\
\text { más altas, así como } \mathrm{T}^{\mathrm{a}} \text { axilar y nivel de } \\
\text { hemoglobina, pero mostró niveles bajos } \\
\text { de sodio en sangre respecto al grupo B. } \\
\text { No se encontraron diferencias } \\
\text { significativas en los niveles de } \mathrm{pH} \\
\text { sanguíneo, la morbilidad neonatal o los } \\
\text { reflejos primitivos en ambos grupos. }\end{array}$ & $\begin{array}{l}\text { Hace referencia a la utilización de } \\
\text { la hidroterapia durante la fase } \\
\text { expulsiva. Por lo tanto, como } \\
\text { existen pocos estudios sobre esta } \\
\text { técnica en la fase expulsiva, es de } \\
\text { gran ayuda para aportar más } \\
\text { evidencia científica y poder aclarar } \\
\text { ciertas dudas existentes todavía en } \\
\text { este campo. }\end{array}$ \\
\hline
\end{tabular}




\begin{tabular}{|c|c|c|c|c|c|}
\hline $\begin{array}{c}\text { The American College of } \\
\text { Obstetricians and } \\
\text { Gynaecologists } \\
2014 \\
\text { EE. UU. }\end{array}$ & $\mathrm{Rb}$. & $\begin{array}{l}\text { Se escogieron } 29 \text { artículos } \\
\text { para la realización de esta } \\
\text { revisión en diferentes bases } \\
\text { de datos. }\end{array}$ & $\begin{array}{l}\text { Identificar los riesgos y } \\
\text { beneficios reportados de la } \\
\text { inmersión en agua durante las } \\
\text { fases de dilatación y la fase } \\
\text { expulsiva del parto. }\end{array}$ & $\begin{array}{l}\text { La inmersión en agua durante la primera } \\
\text { fase del parto puede estar asociada a una } \\
\text { disminución del dolor o uso de analgesia } \\
\text { y a una disminución de la duración del } \\
\text { parto. Sin embargo, no hay evidencia de } \\
\text { que la inmersión durante la fase de } \\
\text { dilatación mejore los resultados } \\
\text { perinatales. Sin embargo, debido a la falta } \\
\text { de información en cuanto a su utilización } \\
\text { en la fase expulsiva y los informes de } \\
\text { casos de efectos adversos poco frecuentes } \\
\text { pero graves en el recién nacido, esta } \\
\text { práctica durante la segunda fase del parto } \\
\text { debe considerarse un procedimiento } \\
\text { experimental que solo debe realizarse en } \\
\text { el contexto de un ensayo clínico con } \\
\text { consentimiento informado. }\end{array}$ & $\begin{array}{l}\text { La inmersión en agua durante la } \\
\text { primera etapa del parto puede estar } \\
\text { asociada con una disminución del } \\
\text { dolor o uso de anestesia y } \\
\text { disminución de la duración del } \\
\text { trabajo de parto. Sin embargo, no } \\
\text { hay evidencia de que la inmersión } \\
\text { durante la primera etapa del trabajo } \\
\text { de parto mejore los resultados } \\
\text { perinatales. En contraste, no se ha } \\
\text { establecido la seguridad y eficacia } \\
\text { de la inmersión en agua durante la } \\
\text { segunda etapa del trabajo de parto. }\end{array}$ \\
\hline $\begin{array}{c}\text { Cluett y Burns } \\
2014 \\
\text { Reino Unido }\end{array}$ & $\mathrm{Rb}$. & $\begin{array}{l}12 \text { ensayos controlados } \\
\text { aleatorios ( } 3243 \text { mujeres } \\
\text { gestantes), en } 8 \text { de las } 12 \\
\text { revisiones las mujeres } \\
\text { utilizaron inmersión en } \\
\text { agua durante la primera fase } \\
\text { del parto, en } 1 \text { inmersión } \\
\text { temprana en comparación } \\
\text { con tardía, en } 2 \text { inmersión } \\
\text { en agua durante la } 2^{\mathrm{a}} \text { y } 3^{\mathrm{a}} \\
\text { fase y en } 1 \text { inmersión } \\
\text { durante la } 2^{\mathrm{a}} \text { fase. }\end{array}$ & $\begin{array}{l}\text { Evaluar la evidencia de ensayos } \\
\text { controlados aleatorios sobre la } \\
\text { inmersión en agua durante la } 1^{\mathrm{a}} \text { y } \\
2^{\mathrm{a}} \text { fase del parto en relación con } \\
\text { los resultados maternos, fetales y } \\
\text { neonatales. }\end{array}$ & $\begin{array}{l}\text { Durante la inmersión en agua en la } 1^{\text {a }} \text { fase } \\
\text { hubo una significante reducción en el uso } \\
\text { de la analgesia epidural y una reducción } \\
\text { del tiempo de duración de dicha fase en } \\
\text { comparación con las mujeres con parto } \\
\text { convencional. No hubo puntuaciones de } \\
\text { Apgar menores de } 7 \text { a los } 5 \text { ' ni tasas de } \\
\text { infecciones neonatales. De los tres } \\
\text { ensayos que compararon la inmersión en } \\
\text { agua durante la } 2^{\mathrm{a}} \text { fase y aquellas que no } \\
\text { utilizaron el agua, solo } 1 \text { mostró un nivel } \\
\text { más alto de satisfacción en su experiencia } \\
\text { de parto. }\end{array}$ & $\begin{array}{l}\text { La inmersión en agua durante la } \\
\text { primera etapa del parto reduce el } \\
\text { uso de analgesia epidural/espinal y } \\
\text { la duración de la primera etapa del } \\
\text { parto. No hay evidencia sobre } \\
\text { efectos adversos para el } \\
\text { feto/neonato o la mujer. }\end{array}$ \\
\hline
\end{tabular}




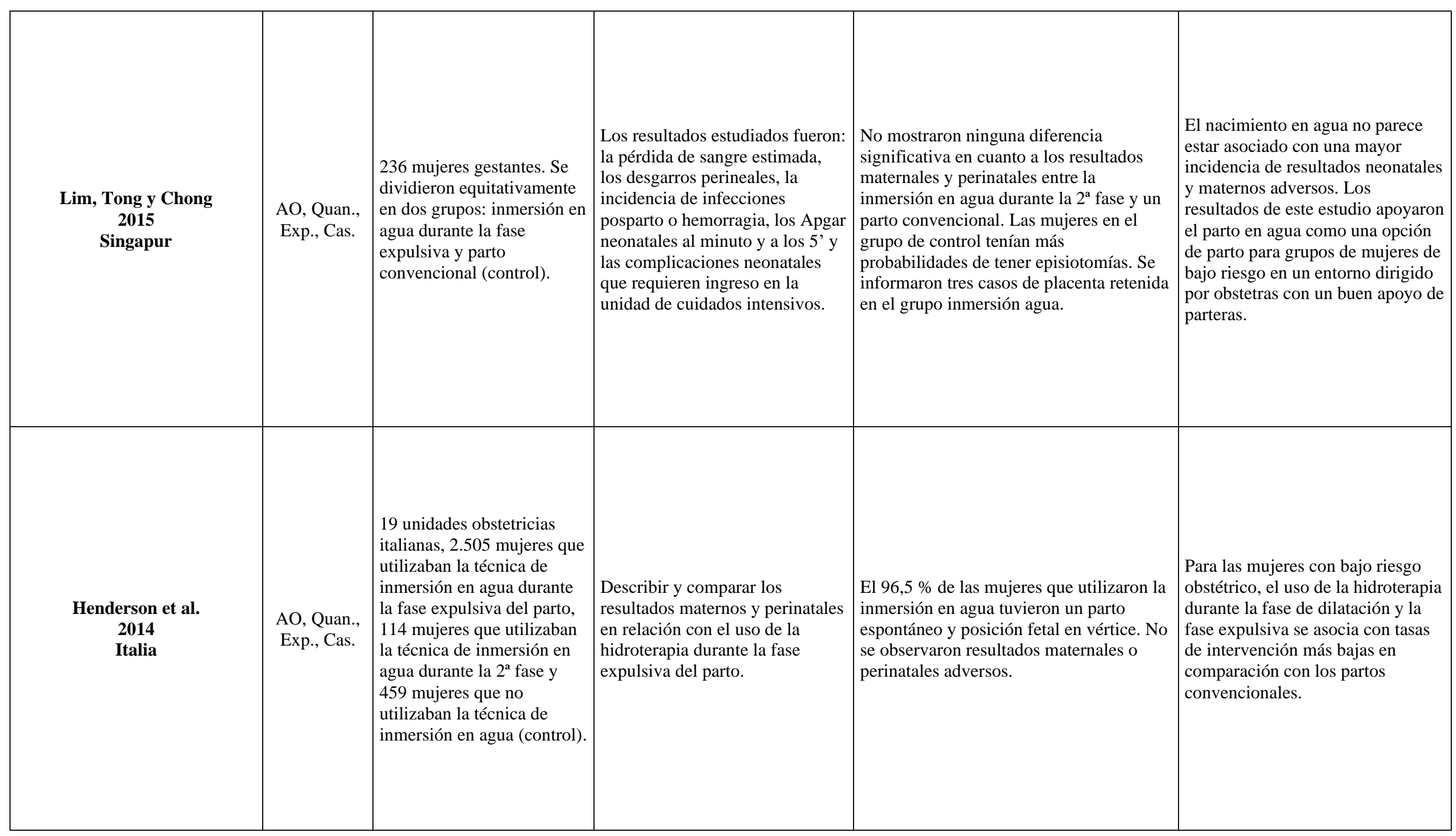




\begin{tabular}{|c|c|c|c|c|c|}
\hline $\begin{array}{c}\text { Torkamani, Kangani y Janani } \\
2010 \\
\text { Irán }\end{array}$ & $\begin{array}{l}\text { AO, Quan., } \\
\text { Exp., Cas. }\end{array}$ & $\begin{array}{l}100 \text { mujeres, divididas } \\
\text { equivalentemente en dos } \\
\text { grupos: uno con parto en el } \\
\text { agua y otro parto } \\
\text { convencional. }\end{array}$ & $\begin{array}{l}\text { Evaluar los efectos de la } \\
\text { hidroterapia en la fase expulsiva, } \\
\text { como un método alternativo de } \\
\text { parto normal sin ningún tipo de } \\
\text { intervención en relación con la } \\
\text { reducción del dolor y duración } \\
\text { del parto. }\end{array}$ & $\begin{array}{l}\text { Reducción significativa en la duración } \\
\text { promedia del parto en aquellos realizados } \\
\text { en el agua y en la duración de la segunda } \\
\text { fase del parto. La medición del dolor en } \\
\text { los dos grupos se realizó mediante la } \\
\text { escala analógica del dolor (EVA) que } \\
\text { indicó una disminución significativa del } \\
\text { dolor en aquellos partos con inmersión } \\
\text { acuática. }\end{array}$ & $\begin{array}{l}\text { El uso de la hidroterapia durante la } \\
\text { fase expulsiva puede llegar a ser } \\
\text { una técnica adecuada, no } \\
\text { farmacológica y no invasiva, } \\
\text { alternativa al parto, ya que reduce } \\
\text { el dolor y la duración del parto. }\end{array}$ \\
\hline $\begin{array}{l}\text { Shaw-Batista } \\
2009 \\
\text { EE. UU. }\end{array}$ & $\begin{array}{l}\text { AO, Quan., } \\
\text { Des., Coh. }\end{array}$ & $\begin{array}{l}13.394 \text { partos llevados a } \\
\text { cabo en el hospital } \\
\text { comunitario de California } \\
\text { durante los primeros } 10 \\
\text { años de la implantación de } \\
\text { la hidroterapia ( } 624 \text { partos } \\
\text { acuáticos durante la fase de } \\
\text { dilatación y } 675 \text { partos } \\
\text { acuáticos durante la fase } \\
\text { expulsiva). }\end{array}$ & $\begin{array}{l}\text { Se compararon la eficacia } \\
\text { perinatal, procesos de atención y } \\
\text { los resultados en tres grupos de } \\
\text { estudio: inmersión en agua } \\
\text { durante la fase de dilatación, } \\
\text { inmersión en agua durante la fase } \\
\text { expulsiva y parto estándar. }\end{array}$ & $\begin{array}{l}\text { El uso de métodos farmacológicos para el } \\
\text { alivio del dolor fue cinco veces mayor en } \\
\text { el grupo sin inmersión en agua en } \\
\text { comparación con el grupo de inmersión } \\
\text { en agua durante la fase expulsiva. La } \\
\text { laceración perineal severa disminuyó en } \\
\text { el grupo de inmersión en agua durante la } \\
\text { fase expulsiva en relación con el grupo de } \\
\text { atención estándar, aunque aumentó la } \\
\text { reparación de la laceración y la laceración } \\
\text { periuretral. La inmersión en agua durante } \\
\text { la fase expulsiva se asoció con una } \\
\text { disminución del aumento del trabajo de } \\
\text { parto, menos antibióticos profilácticos y } \\
\text { terapéuticos y monitorización fetal } \\
\text { intermitente versus continua en } \\
\text { comparación con los grupos sin inmersión } \\
\text { e inmersión en agua durante la fase de } \\
\text { dilatación. No hubo diferencias en los } \\
\text { parámetros maternos o neonatales, } \\
\text { incluidos el método de parto y la } \\
\text { puntuación de Apgar. }\end{array}$ & $\begin{array}{l}\text { La inmersión en agua durante la } \\
\text { fase expulsiva está asociada a } \\
\text { resultados perinatales óptimos y a } \\
\text { menores usos de medicinas y } \\
\text { procesos tecnológicos. Sin } \\
\text { embargo, aumenta la laceración } \\
\text { periuretral. }\end{array}$ \\
\hline
\end{tabular}




\begin{tabular}{|c|c|c|c|c|c|}
\hline $\begin{array}{l}\text { Barbieri et al. } \\
2013 \\
\text { Brasil }\end{array}$ & $\begin{array}{c}\text { AO, Exp., } \\
\text { Quan. }\end{array}$ & $\begin{array}{l}15 \text { mujeres gestantes con } \\
\text { bajo riesgo obstétrico que } \\
\text { aceptaron el uso de } \\
\text { intervenciones no } \\
\text { farmacológicas para el } \\
\text { alivio del dolor. El grupo } 1 \\
\text { recibió como intervención } \\
\text { no farmacológica inmersión } \\
\text { en agua durante la fase de } \\
\text { dilatación, el grupo } 2 \\
\text { realizó ejercicios para el } \\
\text { periné con la bola suiza y el } \\
\text { grupo } 3 \text { inmersión en agua } \\
\text { y balón suizo. }\end{array}$ & $\begin{array}{l}\text { Evaluar, de forma aislada y } \\
\text { combinada, el uso de la } \\
\text { hidroterapia y ejercicios } \\
\text { perineales con el balón suizo } \\
\text { durante la fase de dilatación, en } \\
\text { relación con la percepción del } \\
\text { dolor. La escala visual analógica } \\
\text { (EVA) se utilizó para medir el } \\
\text { dolor antes y } 1 \text { h después de las } \\
\text { intervenciones. Estudio de } \\
\text { evaluación pre-post, (antes y } \\
\text { después). }\end{array}$ & $\begin{array}{l}\text { Se puede observar que, de las } 3 \\
\text { intervenciones, la puntuación de dolor es } \\
\text { menor cuando se relaciona con el uso de } \\
\text { la hidroterapia. }\end{array}$ & $\begin{array}{l}\text { El uso combinado de métodos no } \\
\text { farmacológicos para el alivio del } \\
\text { dolor durante la fase de dilatación, } \\
\text { tales como inmersión en agua y } \\
\text { ejercicios del periné con la bola } \\
\text { suiza, se relacionan con una } \\
\text { reducción del dolor y promueven } \\
\text { el confort de las parturientas. }\end{array}$ \\
\hline $\begin{array}{l}\text { Lee et al. } \\
2013 \\
\text { China }\end{array}$ & $\begin{array}{c}\text { AO, Exp., } \\
\text { Quan. }\end{array}$ & $\begin{array}{l}80 \text { mujeres gestantes: } 41 \text { en } \\
\text { el grupo de control y } 39 \text { en } \\
\text { el grupo experimental. }\end{array}$ & $\begin{array}{l}\text { Determinar la eficacia de la } \\
\text { inmersión en agua en cuanto al } \\
\text { alivio del dolor y la experiencia } \\
\text { de las gestantes durante la } \\
\text { primera fase del parto. Se utilizó } \\
\text { la escala visual analógica del } \\
\text { dolor para evaluar el dolor. }\end{array}$ & $\begin{array}{l}\text { Las mujeres del grupo experimental } \\
\text { informaron de puntuaciones en la escala } \\
\text { visual analógica del dolor más bajas en } \\
\text { las dilataciones cervicales y experiencias } \\
\text { de parto más satisfactorio que en el grupo } \\
\text { de control. }\end{array}$ & $\begin{array}{l}\text { Aparte de los beneficios físicos en } \\
\text { cuanto a la higiene, la inmersión en } \\
\text { agua es una técnica costo efectiva, } \\
\text { conveniente, fácil de desarrollar, y } \\
\text { un método no farmacológico de } \\
\text { alivio del dolor. Esta técnica } \\
\text { propicia la participación constante } \\
\text { de la mujer en todo el proceso de } \\
\text { parto y a obtener experiencias } \\
\text { positivas. }\end{array}$ \\
\hline
\end{tabular}




\begin{tabular}{|c|c|c|c|c|c|}
\hline $\begin{array}{c}\text { Benfield, et al. } \\
2010 . \\
\text { EE. UU.. }\end{array}$ & $\begin{array}{l}\text { AO, Exp., } \\
\text { Quan. }\end{array}$ & $\begin{array}{l}\text { Se reclutaron a gestantes } \\
\text { con bajo riesgo obstétrico } \\
\text { en las clínicas obstétricas } \\
\text { del Departamento de Salud } \\
\text { del Condado, de la Facultad } \\
\text { de Medicina de la } \\
\text { Universidad de Carolina y } \\
\text { de dos consultorios } \\
\text { obstétricos privados. La } \\
\text { muestra resultó ser de } 11 \\
\text { mujeres gestantes. }\end{array}$ & $\begin{array}{l}\text { Se examinaron los efectos de la } \\
\text { hidroterapia en relación con la } \\
\text { ansiedad y dolor materno, las } \\
\text { respuestas endocrinas, el cambio } \\
\text { de volumen plasmático y las } \\
\text { contracciones uterinas durante la } \\
\text { fase de dilatación. En este } \\
\text { estudio no hubo dos grupos } \\
\text { distintos, sino que las gestantes } \\
\text { fueron los propios controles. }\end{array}$ & $\begin{array}{l}\text { Descensos en los niveles de ansiedad, } \\
\text { vasopresina y oxitocina a los } 15 \text { ' y } 45^{\prime} \text { '. } \\
\text { No hubo diferencias significativas entre el } \\
\text { dolor previo a la inmersión y el dolor } \\
\text { durante la inmersión. El dolor disminuyó } \\
\text { más para las mujeres con dolor basal alto. } \\
\text { Durante la inmersión, la frecuencia de las } \\
\text { contracciones uterinas disminuyó. }\end{array}$ & $\begin{array}{l}\text { La hidroterapia durante la fase de } \\
\text { dilatación afecta a las respuestas } \\
\text { endocrinas de nuestro organismo } \\
\text { que modifican procesos } \\
\text { fisiopsicológicos, tales como los } \\
\text { niveles de ansiedad y las } \\
\text { contracciones uterinas. No existen } \\
\text { referencias en cuanto a la } \\
\text { disminución del dolor. }\end{array}$ \\
\hline $\begin{array}{c}\text { Shaw-Battista } \\
\text { 2017. } \\
\text { EE. UU. }\end{array}$ & $\mathrm{Rb}$ & $\begin{array}{l}\text { Se analizaron un total de } 7 \\
\text { artículos. }\end{array}$ & $\begin{array}{l}\text { Se realizó una búsqueda } \\
\text { exhaustiva de artículos en las } \\
\text { bases de datos PubMed y la } \\
\text { Biblioteca Cochrane. Los ítems } \\
\text { seleccionados para este estudio } \\
\text { fueron: alivio del dolor, la } \\
\text { duración del parto, tipo de parto, } \\
\text { laceración obstétrica, satisfacción } \\
\text { materna, puntuación Apgar y } \\
\text { cualquier otro parámetro } \\
\text { perinatal o materno informado. }\end{array}$ & $\begin{array}{l}\text { Se identifican beneficios de la } \\
\text { hidroterapia en cuanto al alivio del dolor e } \\
\text { indican que no hay efectos adversos. Se } \\
\text { observó un alivio de la ansiedad aguda en } \\
\text { aquellas mujeres que utilizaron la } \\
\text { hidroterapia. No se encontraron artículos } \\
\text { que hicieran referencia a efectos dañinos } \\
\text { para la madre o el feto. Tres estudios } \\
\text { demostraron que la hidroterapia promueve } \\
\text { el trabajo fisiológico. No se encontraron } \\
\text { diferencias en cuanto a la duración del } \\
\text { trabajo de parto. No se encontraron } \\
\text { evidencias sobre los beneficios de la } \\
\text { hidroterapia en cuanto a la laceración } \\
\text { obstétrica y el tipo de parto. }\end{array}$ & $\begin{array}{l}\text { Las mujeres con bajo riesgo } \\
\text { obstétrico que deseen utilizar la } \\
\text { hidroterapia durante la fase de } \\
\text { dilatación pueden hacerlo de una } \\
\text { manera segura y como resultado no } \\
\text { precisar de analgesia durante el } \\
\text { parto. Además del alivio del dolor, } \\
\text { existen múltiples mecanismos } \\
\text { adicionales mediante los cuales se } \\
\text { logra un parto lo más fisiológico } \\
\text { posible. }\end{array}$ \\
\hline
\end{tabular}




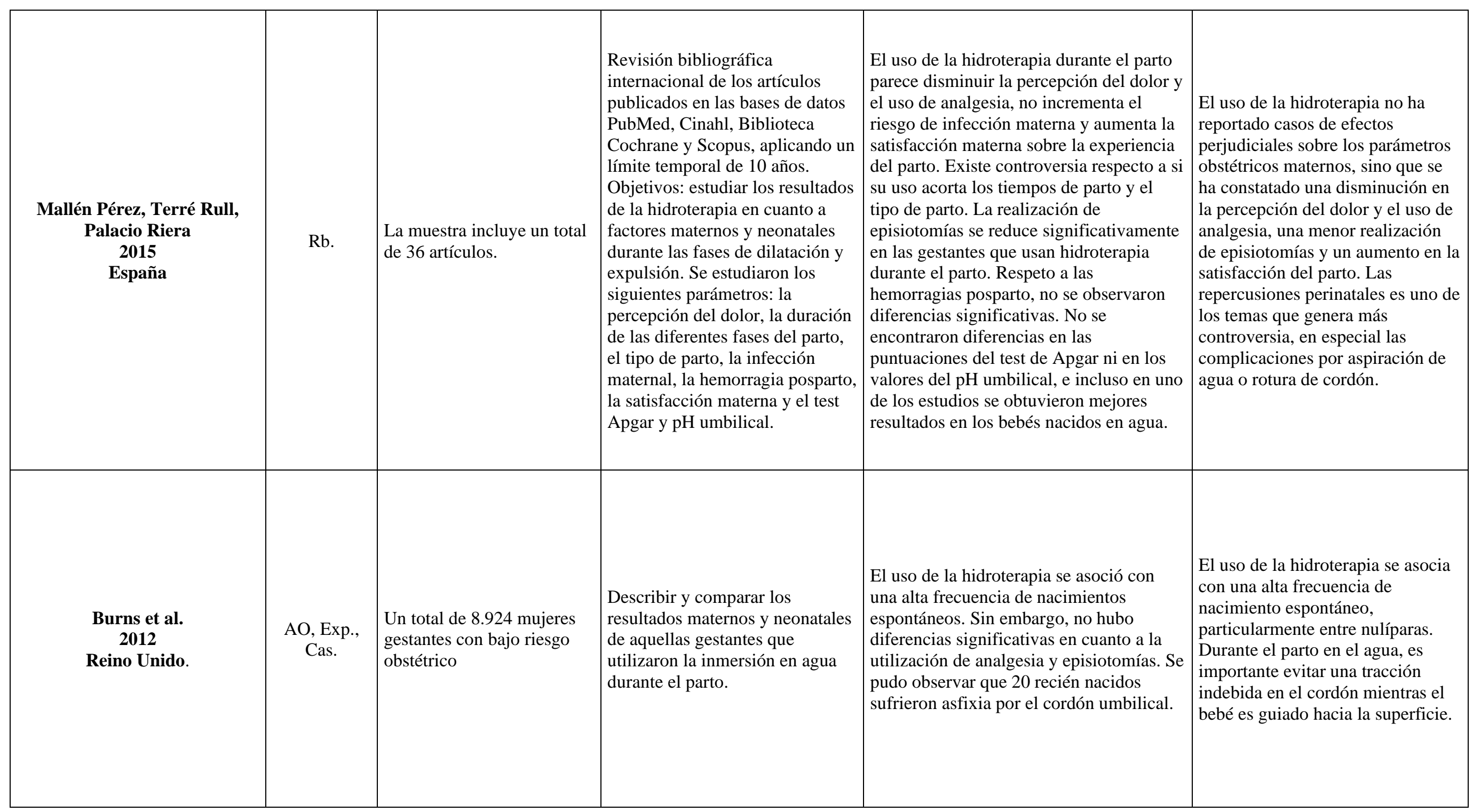




\begin{tabular}{|c|c|c|c|c|c|}
\hline $\begin{array}{c}\text { Mallen Pérez, L. } \\
2017 . \\
\text { España. }\end{array}$ & $\begin{array}{c}\text { AO, Quan., } \\
\text { Quasi. }\end{array}$ & $\begin{array}{l}280 \text { gestantes grupo } \\
\text { hidroterapia }(\mathrm{GH}) \text { o grupo } \\
\text { de control }(\mathrm{GC}) \text { según } \\
\text { deseo y disponibilidad del } \\
\text { uso de la hidroterapia. }\end{array}$ & $\begin{array}{l}\text { Las variables estudiadas fueron: } \\
\text { dolor percibido (mediante escala } \\
\text { numérica del dolor), duración del } \\
\text { parto, tipo de parto, uso o no de } \\
\text { analgesia, estado perineal } \\
\text { posparto y la satisfacción } \\
\text { materna mediante la escala de } \\
\text { satisfacción de Mackey. Los } \\
\text { resultados perinatales se } \\
\text { evaluaron con el test Apgar, pH } \\
\text { cordón umbilical y valoración } \\
\text { registro cardiotocográfico. }\end{array}$ & $\begin{array}{l}\text { La percepción del dolor fue } \\
\text { significativamente menor en GH respeto } \\
\text { al GC, se identificó una disminución en el } \\
\text { tiempo de dilatación significativa, no } \\
\text { siendo así en la fase expulsiva y el } \\
\text { promedio total, y una reducción en el uso } \\
\text { de la analgesia. No se observaron } \\
\text { diferencias estadísticamente significativas } \\
\text { en el tipo de parto ni en el estado perineal } \\
\text { postparto. Se observó que el grupo GH } \\
\text { mostraba una satisfacción mayor al GC. } \\
\text { En relación con los resultados perinatales, } \\
\text { no se observaron diferencias en ninguno } \\
\text { de los ítems estudiados. En los neonatos } \\
\text { nacidos bajo el agua, se observaron unos } \\
\text { resultados del test de Apgar al minuto } \\
\text { significativamente menores respeto al } \\
\text { grupo control. }\end{array}$ & $\begin{array}{l}\text { El uso de la hidroterapia durante el } \\
\text { parto es efectiva para disminuir la } \\
\text { percepción del dolor durante el } \\
\text { trabajo de parto y por lo tanto una } \\
\text { consiguiente disminución en los } \\
\text { requerimientos de analgesia. } \\
\text { Además, disminuye el tiempo de } \\
\text { dilatación sin modificar el tiempo } \\
\text { expulsivo. En cuanto a los } \\
\text { resultados perinatales, no se } \\
\text { observan efectos secundarios } \\
\text { destacables, con lo que se } \\
\text { comprueba la seguridad de su uso } \\
\text { para el recién nacido. }\end{array}$ \\
\hline
\end{tabular}


Tabla 4. Resultados en relación con las percepciones de las enfermeras y las gestantes

\begin{tabular}{|c|c|c|c|c|c|}
\hline Autor, Año, País & $\begin{array}{c}\text { Tipo } \\
\text { artículo/ } \\
\text { Diseño }\end{array}$ & Sujetos/Ámbito & $\begin{array}{c}\text { Variables/Dimensiones } \\
\text { /Instrumentos/ } \\
\text { Intervención }\end{array}$ & Resultados & $\begin{array}{l}\text { Conclusiones/Implicaciones } \\
\text { práctica/Investigación }\end{array}$ \\
\hline $\begin{array}{c}\text { Lewis, et al. } \\
2016 \\
\text { Australia }\end{array}$ & $\begin{array}{l}\text { AO, Quali., } \\
\text { Exp. }\end{array}$ & $\begin{array}{l}342 \text { mujeres que firmaron } \\
\text { el consentimiento del uso } \\
\text { de la hidroterapia, de las } \\
\text { cuales } 296 \text { acordaron } \\
\text { participar en el estudio y } \\
\text { un total de } 93 \text { mujeres } \\
\text { realizaron un parto } \\
\text { acuático. }\end{array}$ & $\begin{array}{l}\text { Explorar las } \\
\text { percepciones y } \\
\text { experiencias de las } \\
\text { mujeres que lograron un } \\
\text { parto acuático. }\end{array}$ & $\begin{array}{l}\text { Los resultados mostraron los siguientes } \\
\text { motivos por los cuales las mujeres se } \\
\text { decidían por la utilización de la hidroterapia } \\
\text { durante el parto: disminución del dolor, les } \\
\text { gustaba la idea porque querían un parto lo } \\
\text { más natural posible, porque lo percibieron } \\
\text { como un ambiente de relajación y, por } \\
\text { último, porque se lo habían recomendado. } \\
\text { Sin embargo, el factor principal por el cual } \\
\text { se decantaban todas las mujeres gestantes } \\
\text { fue la reducción del dolor. }\end{array}$ & $\begin{array}{l}\text { La inmersión en agua aporta un cambio en el } \\
\text { enfoque en el cuidado obstétrico. Además, } \\
\text { aporta evidencia científica y un cuidado } \\
\text { obstétrico respetuoso por parte de las matronas, } \\
\text { que a su vez da lugar a que las gestantes } \\
\text { puedan obtener una experiencia agradable de } \\
\text { su parto. }\end{array}$ \\
\hline $\begin{array}{c}\text { Stark y Miller } \\
2010 \\
\text { EE. UU. }\end{array}$ & $\begin{array}{l}\text { AO, Quali., } \\
\text { Exp. }\end{array}$ & $\begin{array}{l}\text { Después de una revisión } \\
\text { de la literatura y de } \\
\text { entrevistas con } 10 \\
\text { enfermeras, } 2 \text { comadronas } \\
\text { y } 6 \text { enfermeras } \\
\text { investigadoras cuya } \\
\text { experiencia consistía en } \\
\text { brindar atención a las } \\
\text { mujeres gestantes. El } \\
\text { cuestionario fue facilitado } \\
\text { a } 65 \text { enfermeras } \\
\text { comadronas y a } 401 \\
\text { enfermeras generalistas. }\end{array}$ & $\begin{array}{l}\text { Desenrollar y testar un } \\
\text { instrumento sobre la } \\
\text { percepción de las } \\
\text { enfermeras en cuanto a } \\
\text { las barreras que existen } \\
\text { en el uso de la } \\
\text { hidroterapia durante el } \\
\text { trabajo de parto. } \\
\text { Después de las } \\
\text { entrevistas a las } 18 \\
\text { enfermeras, los } \\
\text { investigadores } \\
\text { adquirieron los ítems } \\
\text { para generar el } \\
\text { cuestionario, revisado } \\
\text { posteriormente por } 4 \\
\text { expertos. }\end{array}$ & $\begin{array}{l}\text { Las preocupaciones de las enfermeras } \\
\text { fueron: 1. Conocimientos y creencias, que } \\
\text { incluyen aspectos como la seguridad y la } \\
\text { eficacia de esta técnica. 2. Entorno de los } \\
\text { cuidados enfermero en relación con } \\
\text { elementos propios de la cultura de los } \\
\text { centros de maternidad. 3. Preocupaciones } \\
\text { personales sobre incomodidades y riesgos } \\
\text { asociados con la hidroterapia. 4. Esfuerzo } \\
\text { físico requerido para la técnica de la } \\
\text { hidroterapia, especialmente en relación con } \\
\text { el tiempo y el esfuerzo necesarios para el } \\
\text { parto. 5. Tecnología, en relación con el } \\
\text { control del feto, la responsabilidad legal y } \\
\text { el acceso a las intervenciones. }\end{array}$ & $\begin{array}{l}\text { Promocionar la hidroterapia durante la fase de } \\
\text { dilatación requiere que todas las enfermeras } \\
\text { tengan específicos conocimientos y habilidades } \\
\text { sobre el tema. Un entorno de apoyo en los } \\
\text { cuidados de enfermería que incluya tanto a } \\
\text { otras enfermeras como a administración es de } \\
\text { gran ayuda para la promoción de la } \\
\text { hidroterapia. Cuando existen barreras en cuanto } \\
\text { al uso de esta técnica, la hidroterapia requiere } \\
\text { mayores esfuerzos y su implementación es } \\
\text { difícil. En consecuencia de lo anterior, las } \\
\text { enfermeras no ofrecerán o, por el contrario, } \\
\text { promoverán el uso de esta terapia } \\
\text { complementaria. }\end{array}$ \\
\hline
\end{tabular}




\section{DISCUSIÓN}

\section{Efectos de la hidroterapia durante la fase de dilatación}

En un estudio llevado a cabo por Shu-Ling Lee et al. (2013), en la sala de maternidad de un hospital de la ciudad de Taipei, se concluyó que las mujeres que utilizaron la hidroterapia durante la fase de dilatación informaron de puntuaciones de EVA (Escala Analgésica del Dolor) significativamente más bajas durante las dilataciones cervicales y mayores experiencias de parto positivas. Por otro lado, un ensayo clínico a ciegas realizado en la ciudad de Sao Paulo, en un centro de partos naturales, en el año 2013, el cual buscaba comprobar la eficacia de la hidroterapia, los ejercicios perineales y el uso de la pelota suiza durante la fase de dilatación para el alivio del dolor, concluyó afirmando que, cuando se utilizaban las dos técnicas individualmente, los valores no indicaban ninguna diferencia significativa en cuanto a la puntuación del dolor. Sin embargo, de entre las tres técnicas, la hidroterapia durante la fase de dilatación anotaba una puntuación más baja de dolor.

Además, los valores sugirieron que el uso combinado de la hidroterapia y la utilización de la pelota suiza reducían significativamente la puntuación del dolor (Bellido y Lendínez, 2010). Cluett y Burns (2011) afirmaron que la inmersión en agua durante la primera fase del parto reduce el uso de la analgesia epidural y la duración de esta fase y que existe información limitada de otros resultados sobre el uso de la hidroterapia en la primera y segunda fase del parto. También identificaron que existe un déficit de evidencia científica sobre efectos adversos en el feto o de las parturientas con la utilización de la hidroterapia en las dos fases del parto. Sin embargo, en otro estudio realizado por Benfield et al. (2010), la hidroterapia durante la fase de dilatación se asoció a descensos en los niveles de ansiedad, pero solo se referencia disminución del dolor en aquellas mujeres con nivel basal de dolor alto. Por otro lado, la American Academy of Pediatrics junto al American College of Obstetricians and Gynecologists (2014) elaboraron un informe clínico sobre los riesgos y los beneficios de la inmersión en el agua durante la fase de dilatación y la fase expulsiva. Después de revisar la bibliografía escrita sobre este tema, se concluyó que no hay evidencia científica sobre los beneficios de la inmersión en agua durante la fase de dilatación, aunque sí que mejora los resultados perinatales. 
En dos estudios realizados por Shaw-Battista (2017) y Burns et al. (2012), no se especifica si los resultados obtenidos hacen referencia a la hidroterapia utilizada durante la primera o segunda fase del parto, pero se evidencia una disminución del dolor y de la ansiedad y dicha técnica se asoció con una alta frecuencia de nacimientos espontáneos sin que se identificaran resultados adversos para la madre o el bebé.

\section{Efectos de la hidroterapia durante la fase expulsiva}

Según el estudio citado, realizado por Shaw-Batista (2017) en un hospital comunitario de California, el uso de medidas farmacológicas para el alivio del dolor fue mayor en aquellas gestantes que no realizaron la inmersión en agua que en aquellas que, por el contrario, sí que la utilizaron. Además, disminuyó la laceración perianal severa, la administración de antibióticos profilácticos y terapéuticos y la monitorización fetal intermitente. Sin embargo, hubo un aumento de la laceración periuretral y la necesidad de reparación de dicha laceración. Asimismo, Mallen, Terré y Palacio (2015), concluyeron que el uso de la hidroterapia durante el nacimiento no parece afectar a las puntuaciones del test Apgar, así como a los valores de $\mathrm{pH}$ de cordón umbilical, e incluso en algunos casos se obtuvieron mejores resultados en los bebés nacidos en agua.

Los autores Mollamahmutoglu et al, (2012) y Torkamani et al. (2010), en sus respectivos estudios, también demostraron que el uso de la hidroterapia durante la fase expulsiva acorta su duración y también contribuye a la disminución del tiempo en la tercera fase. Además, las gestantes precisaron de menos requerimientos de analgesia y la puntuación del dolor disminuyó, aunque aumentó la laceración perianal. Por otro lado, los autores Lim et al. (2016) y Henderson (2014), no identificaron resultados maternales y perinatales adversos en su estudio. A pesar de estos resultados, la revisión realizada por la American Academy of Pediatrics y el American College of Obstetricians and Gynecologists (2014) señaló que la seguridad y la eficacia de la inmersión en agua en la fase expulsiva no habían sido establecidas y que no se habían asociado beneficios maternales o fetales claros asociados al uso de esta técnica. Según estas instituciones, por estos motivos, junto a los casos reportados sobre efectos adversos en el recién nacido, la práctica de la inmersión en agua en la fase expulsiva debería ser considerada un procedimiento experimental en el contexto de un ensayo clínico.

Del mismo modo, Mallén realizó un estudio multicéntrico cuasi experimental en 2017 en el cual se pudo constatar que, en relación a los resultados neonatales, no se hallaron 
diferencias estadísticamente significativas; solo se constató que el Test de Apgar de los neonatos nacidos bajo el agua al minuto fue significativamente menor respeto al grupo control. Sin embargo, por otro lado, sí que detectó que el uso de la hidroterapia durante el parto disminuye el dolor percibido y los requerimientos de analgesia, así como el tiempo de dilatación, y que mejora el estado perineal posparto y la satisfacción materna, con ausencia de efectos adversos para la gestante. Ros identificó que no se encontraron diferencias significativas en los niveles de $\mathrm{pH}$ sanguíneo, la morbilidad neonatal y los reflejos primitivos de los recién nacidos en agua, pero sí que se observaron puntuaciones de Apgar más altas.

\section{Percepciones de matronas y gestantes en relación con el uso de la hidroterapia}

En un estudio realizado Stark y Miller en 2010, en el cual se distribuyeron cuestionarios sobre la percepción del uso de la hidroterapia a un total de 401 enfermeras y matronas, se observaron cinco dimensiones diferentes sobre las cuales las matronas se preocupaban a la hora de fomentar el uso de la hidroterapia: creencias y conocimientos, el entorno de los cuidados de salud, preocupaciones personales, el esfuerzo requerido por parte de las enfermeras y matronas durante el uso de la hidroterapia y, por último, la tecnología. En la dimensión de creencias y conocimientos, las enfermeras hacían hincapié en la seguridad y la eficacia de dicha técnica, mientras que la segunda trataba sobre la cultura de los centros de maternidad en relación a aspectos tales como los proveedores de salud, los profesionales sanitarios, gestión enfermera, políticas y procedimientos de la hidroterapia y accesibilidad a la utilización de esta técnica. La tercera dimensión contenía subdimensiones sobre la incomodidad y riesgos asociados a la hidroterapia.

Por su parte, en la penúltima dimensión, las preocupaciones de las enfermeras eran sobre el tiempo y esfuerzo dedicado al parto cuando la hidroterapia es proporcionada a las parturientas. En la última, la tecnología, se identificaron factores tales como la monitorización fetal. Por último, se concluyó que aquellas enfermeras que ejercían en un hospital previsto con bañeras disponibles para las parturientas tenían más predisposición al uso de la hidroterapia. Asimismo, el estudio de Mallen (2015) percibió una mejora de la satisfacción materna con el uso de la hidroterapia. No se pudieron encontrar otros artículos que estudiaran diferentes percepciones de enfermeras y matronas. Solo se encontró un único artículo que analizara las motivaciones de las gestantes. En este 
artículo, de Lewis et al (2018), se identifican las razones por las cuales las gestantes deciden tener un parto acuático, siendo el alivio del dolor la razón que tiene más.

\section{CONCLUSIONES}

La mayoría de los estudios realizados sobre el uso de la hidroterapia durante el trabajo de parto no hacen distinción entre su uso durante la fase de dilatación o durante la fase expulsiva del parto. Los resultados maternales y perinatales pueden llegar a ser muy diferentes dependiendo de en qué fase se utiliza la hidroterapia. Por lo tanto, es esencial que las futuras líneas de investigación hagan hincapié en diferenciar su uso durante una fase u otra. Su uso durante la fase de dilatación aporta una mayor satisfacción para la mujer gestante y disminuye el dolor y la necesidad de utilizar medidas farmacológicas para aliviarlo. No obstante, hay pocos estudios que avalen unos buenos resultados, tanto maternales como perinatales, si se utiliza durante la fase expulsiva. En consecuencia, se considera que la utilización de la hidroterapia durante esta fase debería ser meramente científica y bajo supervisión, ya que puede llegar a ser dañino para la madre y/o el feto.

A pesar de los pocos estudios concluyentes sobre los beneficios de utilizar la hidroterapia durante la fase de dilatación, muchos hospitales, tanto internacionales como nacionales, disponen de bañeras para aquellas gestantes que deseen utilizarlas, y son muchas las mujeres que solicitan este tipo de medida no farmacológica, ya que han oído hablar sobre su principal beneficio: el alivio del dolor. Sin embargo, es un tema que genera controversia y muchas asociaciones no están a favor de uso y, por lo tanto, no lo promueven ni a las gestantes ni a las propias profesionales, que al fin y al cabo son las que proporcionan apoyo y ayudan a la mujer durante su parto.

Existen pocos estudios que investiguen sobre la percepción subjetiva de la satisfacción con el uso de la hidroterapia por parte de mujeres gestantes y profesionales sanitarios (matronas), por lo que sería deseable mayor investigación en este sentido. 


\section{BIBLIOGRAFÍA}

BARBIERI, M., et al. Banho quente de aspersão, exercícios perineais com bola suíça e dor no trabalho de parto. En: Acta Paulista de Enfermagem. 2013, vol. 26, núm. 5, pp. $478-484$.

Bellido, J.; J. LendínEZ. Proceso enfermero desde el modelo de cuidados de Virginia Henderson y los lenguajes NNN. Jaén: Colegio Oficial de Enfermería de Jaén, 2010. [Consulta: 11 de enero de 2018]. Disponible en:

〈www.index-f.com/lascasas/documentos/lc0714.pdf $>$.

BENFIELD, R. D., et al. The effects of hydrotherapy on anxiety, pain, neuroendocrine responses, and contraction dynamics during labor. En: Biol Res Nurs. 2010, vol. 12, núm. 1, pp. 28-36.

BuRns, E., et al. Characteristics, Interventions and Outcomes of Women Who Used a Birthing Pool: A Prospective Observational Study. En: Birth. 2012, vol. 39, núm. 3, pp. 192-202.

CluetT, E. R.; E. BuRns. Immersion in water in labour and birth. En: Cochrane Database of Systematic Reviews. 2009, vol. 15, núm. 2. Disponible en:

<dx.doi.org/10.1002/14651858.CD000111.pub3 >

Federación de Asociaciones de Matronas de España (FAME). Iniciativa Parto Normal. Documento de Consenso. [Consulta: 8 de enero de 2018]. Disponible en: <orbananos.files.wordpress.com/2008/03/ipn_libro.pdf>.

FEDERACIÓn DE ASOCIACIONES DE MATRONAS DE EsPaÑa (FAME). Nota de prensa: La evidencia científica lo avala: el parto en casa es seguro. Barcelona: FAME, 2017.

Generalitat de Catalunya. Departament De Salut. Tretze hospitals de la xarxa pública de Catalunya disposen de banyera per oferir atenció al part normal. Barcelona: Generalitat de Catalunya, 2013. [Consulta: 11 de enero de 2018]. Disponible en:

<premsa.gencat.cat/pres_fsvp/AppJava/notapremsavw/197128/ca/hospitals-xarxapublica-catalunya- disposen-banyera-oferir-atencio-part-normal.do> .

HENDERSON, J., et al. Labouring women who used a birthing pool in obstetric units in Italy: prospective observational study. En: BMC Pregnancy and Childbirth. 2014, vol. 14, núm. 17. 
LEE, S., et al. Efficacy of Warm Showers on Labor Pain and Birth Experiences During the First Labor Stage. En: Journal of Obstetric, Gynecologic \& Neonatal Nursing. 2013, vol. 42, núm. 1, pp. 19-28

LEWIS, L., et al. The perceptions and experiences of women who achieved and did not achieve a waterbirth. En: BMC Pregnancy and Childbirth. 2018, vol. 18, núm. 1.

LiM, K.; P. Tong; Y. ChOng. A comparative study between the Pioneer cohort of waterbirths and conventional vaginal deliveries in an obstetrician-led unit in Singapore. En: Taiwanese Journal of Obstetrics and Gynecologists. 2016, vol. 55, núm. 3, pp. 363-367.

Mallén-PÉRez, L.; M. E. JuvÉ-UdinA; M. T. RoÉ-JustiniAno. Dolor de parto: análisis del concepto. En: Matronas Prof. 2015, vol. 16, núm. 2, pp. 61-67.

MalléN, L.; C. Terré; M. PALACiO. Inmersión en agua durante el parto: revisión bibliogràfica. En: Matronas Prof. 2015, vol. 16, núm. 3, pp. 108-113.

MALlÉn PÉREZ, L. Efectividad del uso de la hidroterapia en el trabajo de parto. [Tesis doctoral]. Barcelona: Universidad de Barcelona, 2017.

Ministerio de SANIDAd y POLÍTICA Social. Guía de Práctica Clínica sobre la Atención al Parto Normal.Madrid: Ministerio de Sanidad y Consumo, 2010. [Consulta: 3 de enero de 2018]. Disponible en:

<www.msps.es/organozacion/sns/planCalidadSNS/atencionParto.htm>.

MollamahmutoĞLu, L., et al. The effects of immersion in water on labor, birth and newborn and comparison with epidural analgesia and conventional vaginal delivery. En: J Turkish Ger Gynecol Assoc. 2012, vol. 13, núm. 1, pp. 45-49.

NICHOLLS, S., et al. Exploring midwives; perception of confidence around facilitating water birth in Western Australia: A qualitative descriptive study. En: Midwifery. 2016, vol. 33, pp. 73-81.

ODENT, M. El agua como medio en el parto. En: Natura Med. 1999, vol. 55, pp. 37-41.

ORGANIZACIÓN MUNDIAL De LA SALUD. Cuidados en el parto normal: una guía práctica. Grupo técnico de trabajo de la OMS. Departamento de Investigación y Salud Reproductiva. Ginebra: OMS, 1996. [Consulta 4 de enero de 2018]. Disponible en: < http://comatronas.es/contenidos/2012/03/textos/Guia_practica_de_cuidados_en_el _parto_normal_OMS.pdf>. 
Royal College Of Midwives. Immersion in Water during Labour and Birth. London: RCM, 2009. [Consulta: 10 de enero de 2018]. Disponible en: <www.rcm.org.uk/sites/default/files/Immersion\%20in\%20Water\%20\%20for\%20La bour\%20and\%20Birth_0.pdf>.

Ros, H. Effects of aterbirths and traditional bedbirths on outcomes for neonates. En: Curationis. 2009, vol. 32, núm. 2, pp. 46-52.

SHAW-BATTISTA, J. Optimal outcomes of labor and birth in water compared to standard maternity care [Tesis doctoral]. San Francisco: University of California, 2009.

Shaw-BatTista, J. Systematic Review of Hydrotherapy Research. En: The Journal of Perinatal \& Neonatal Nursing. 2017, vol. 31, núm. 4, pp. 303-316.

Sociedad Española De Obstetricia Y Ginecología (SEGO). Recomendaciones sobre la asistencia al parto. Madrid: SEGO, 2008. [Consulta: 3 de enero de 2018]. Disponible en:

<www.juntadeandalucia.es/servicioandaluzdesalud/hinmaculada/web/servicios/tcg/ documentos/Protocolos/Guias\%20Practica\%20Clinica/PartoNormal08.pdf>.

Stark, M.; M. MiLler. Development and Testing of Nurses' Perceptions of the Use of Hydrotherapy in Labor Questionnaire. En: Journal of Nursing Measurement. 2010, vol. 18 , núm. 1 , pp. 36-48.

ShU-Ling, L., et al. Efficacy of Warm Showers on Labor Pain and Birth Experiences During the First Labor Stage. En: JOGNN. 2013, vol. 42, pp. 19-28. [Consulta: Enero de 2018]. Disponible en: 〈www.jognn.org/article/S0884-2175(15)31247-8/pdf〉 .

The American ACADEmy of Pediatrics; The American College of Obstetricians AND GYNECOLOGISTS. Immersion in Water During Labor and Delivery. En: Pediatrics. 2014, vol. 133, núm. 4, pp. 758-761.

TORKAMAni, S. A.; F. KANGANI; F. JANANI. The effects of delivery in water on duration of delivery and pain compared with normal delivery. En: Pak J Med Sci. 2010, vol. 26, núm. 3, pp. 551-555. 